\title{
Método para la predicción de la dinámica temporal de la malaria en los municipios de Colombia
}

\author{
Javier Oswaldo Rodríguez Velásquez ${ }^{1}$
}

Forma de citar

Rodríguez Velázquez JO. Método para la predicción de la dinámica temporal de la malaria en los municipios de Colombia. Rev Panam Salud Publica. 2010;27(3):211-8.

RESUMEN Objetivo. Desarrollar una metodología para la predicción de la dinámica temporal de los brotes de malaria en los municipios de Colombia.

Métodos. Se definieron rangos epidemiológicos, definidos por los múltiplos de 50 casos para el grupo de seis municipios con mayor incidencia, de 25 casos para los municipios que ocuparon las posiciones 10 y 11 por su incidencia, de 10 para el municipio que ocupó la posición 193 y de 5 para el municipio que ocupó la posición 402. Se calcularon los valores de probabilidad específica de la aparición de cada rango epidemiológico en cada municipio, así como el valor de $S / k$ - como la relación entre la entropía (S) y la constante de Boltzmann ( $k$ ) - para cada grupo de tres semanas y las restas de esta relación entre los grupos consecutivos de semanas. Con estas relaciones matemáticas se establecieron los valores de predicción de la dinámica de casos y se compararon con los datos epidemiológicos reales en el período 2003-2007.

Resultados. La probabilidad de aparición de los rangos epidemiológicos varió entre 0,019 y 1,00, mientras que las restas de la relación $S / k$ de grupos de semanas consecutivas variaron entre $-0,23$ y 0,29. Se establecieron tres relaciones que determinan si la dinámica corresponde a un brote. Estas relaciones se confirmaron con los datos epidemiológicos reales de los $810 \mathrm{mu}$ nicipios colombianos.

Conclusiones. Esta metodología permite predecir la dinámica de los casos y los brotes de malaria en los municipios de Colombia y se pueden aplicar en la planificación de intervenciones y políticas de salud pública.

Palabras clave Malaria; predicción; distribución temporal; brotes de enfermedades; vigilancia epidemiológica; Colombia.

Según datos de la Organización Mundial de la Salud, la malaria afecta de 350 a 500 millones de personas en todo el mundo y alrededor de 1 millón de ellas muere anualmente a causa de esta enfer-

\footnotetext{
Grupo de Investigación Insight, Centro de Investigaciones de la Clínica del Country, Bogotá, Colombia. La correspondencia se debe dirigir al autor a Grupo Insight, Cra. 79b No. 51-16 sur, interior 5, apartamento 102, Bogotá, Colombia. Correo electrónico: grupoinsight2025@yahoo.es, grupo insight@yahoo.es
}

medad (1). De los 108 países en los que la malaria está reconocida como una enfermedad endémica, en 38 países -9 de ellos africanos- se logró reducir en 2008 el número de casos de malaria a menos de la mitad de los registrados en 2000. Esta reducción, sin embargo, fue menor en los países que tienen las mayores tasas de incidencia (1).

En Colombia, alrededor de 25 millones de personas están en riesgo de contraer la malaria, transmitida principal- mente por los parásitos Plasmodium falciparum y $P$. vivax. A pesar de las medidas de control implementadas, el índice anual de incidencia parasitaria ha mostrado una tendencia a aumentar a partir de 2006, por lo que la malaria constituye una preocupación actual de salud pública nacional. Esta situación es especialmente preocupante en las regiones con mayor riesgo de transmisión, como las franjas costeras del Pacífico y el golfo de Urabá, las cuencas de los ríos Cauca bajo 
y Sinú alto y los territorios de Orinoquia y Amazonia (2).

La vigilancia epidemiológica tiene como objetivo detectar tempranamente aumentos en el número de casos de una enfermedad dada, para lo que es fundamental conocer los niveles habituales de incidencia en cada población y momento del año. También para la vigilancia de los brotes -definidos como un aumento abrupto en el número de casos en una población, un período o un sitio geográfico dados- es necesario conocer la dinámica habitual de casos de nuevo diagnóstico. El establecimiento de canales o corredores endémicos de una enfermedad puede contribuir a definir los valores de incidencia esperados en una dinámica específica, lo que permitiría evidenciar la aparición de brotes (3).

Algunos estudios han relacionado los brotes de malaria con algunos factores climáticos y biológicos, como la calidad y la distribución del agua superficial, los sitios de reproducción de los insectos vectores (4), la temperatura, la humedad $(4,5)$ y las variaciones en los ecosistemas de predadores de insectos $(6,7)$, entre otros. Algunos autores afirman que los picos de algunas enfermedades transmitidas por vectores cuyas larvas se desarrollan en el agua están relacionados con anomalías climáticas producidas por eventos como El Niño/Oscilación del Sur. Aumentos en el número de casos de malaria en Pakistán (8), Venezuela (9) y Colombia $(10,11)$ se han asociado con esta anomalía. Sin embargo, este tipo de correlaciones con el clima son de utilidad limitada, dado que resultan difíciles de extrapolar en el espacio y el tiempo y no se pueden generalizar fácilmente.

Algunas leyes y teorías matemáticas y físicas aplicadas a diferentes fenómenos en medicina han permitido desarrollar medidas objetivas que ayudan a comprender mejor su dinámica. Así, con el método probabilístico de la caminata al azar, basado en la dinámica geométrica del número de casos anuales registrados, se han podido hacer predicciones temporales de brotes de dengue (12) y malaria en Colombia, así como de la infección por el VIH en los Estados Unidos de América (datos no publicados). Estas predicciones se corroboraron con los datos del Instituto Nacional de Salud de Colombia e instituciones estadounidenses, con valores de precisión de 90,4\%, $95,60 \%$ y $99,7 \%$, respectivamente.

A partir de la probabilidad se puede cuantificar la posibilidad de que ocurra un evento (13). Si bien algunos sistemas complejos basados en una gran cantidad de información - como la dinámica de una epidemia- se pueden evaluar como sistemas probabilísticos, para determinar el comportamiento dinámico de este tipo de sistemas es necesario utilizar los conceptos de entropía (S), la constante de Boltzmann (k) y la relación de ambos $(\mathrm{S} / \mathrm{k})$, originalmente desarrollados para estudios termodinámicos y de mecánica estadística. El concepto de entropía permite abordar el número de configuraciones microscópicas que puede presentar un sistema en estado de equilibrio - como las múltiples moléculas de un gas - para que siga siendo el mismo desde el punto de vista macroscópico. Por su parte, la relación S/k permite simplificar los resultados al dividirlos por la constante de Boltzmann, lo que evita llegar a valores exponenciales en la ecuación original (14). En este contexto, la entropía se definió inicialmente para sistemas equiprobables —cuando los eventos tienen la misma probabilidad de presentarse - como una medida proporcional a la cantidad de diferentes configuraciones en las que se puede presentar un estado macroscópico en un sistema. Posteriormente, este concepto se generalizó en la mecánica estadística para sistemas fuera de equilibrio, lo que determinó la entropía de sistemas no equiprobales como una medida proporcional a la suma de las probabilidades multiplicadas por su logaritmo (14-15). Este concepto ha encontrado múltiples aplicaciones fuera del campo de la mecánica. Por ejemplo, en el contexto de la teoría de la información, Shannon encontró que este enfoque permite cuantificar la cantidad de información que contiene un mensaje como una medida matemática de la cantidad de información que posee un sistema (16).

Como se sabe, la malaria presenta un comportamiento complejo y prácticamente impredecible, por lo que se podrían utilizar la teoría de la probabilidad, el concepto de entropía según Boltzmann-Gibbs y su relación $S / k$ para cuantificar la dinámica de los casos de malaria de manera objetiva y reproducible desde una perspectiva acausal. De esta forma se podría realizar una predicción en el espacio y el tiempo de los brotes a partir de la cuantificación de los cambios semanales de la dinámica de casos.

El objetivo del presente trabajo fue desarrollar una metodología para la predic- ción de la dinámica temporal de los brotes de malaria en los municipios de Colombia que pueda utilizarse como base de un sistema de vigilancia.

\section{MATERIALES Y MÉTODOS}

Se utilizó la base de datos del Sistema de Vigilancia y Alerta Temprana (SIVIGILA) del Instituto Nacional de Salud de Colombia para el período 2003-2007 y los registros del número de infectados con malaria en cada una de las 53 semanas epidemiológicas de los años estudiados y cada uno de los 820 municipios de Colombia. A pesar de cierto grado de subregistro (2), estos datos son los de mayor fiabilidad, ya que el Instituto Nacional de Salud de Colombia registra todos los casos positivos a la prueba de la gota gruesa notificados en el país.

Se seleccionaron 10 municipios, separados en cuatro grupos según el número total de personas infectadas durante el período 2003-2007. El grupo A quedó compuesto por los seis municipios con mayor número de casos $(34,6 \%$ del total de infectados en el país en el período 2003-2007), el grupo B con los municipios que ocuparon las posiciones 10 y 11 por su número de infectados $(4,4 \%$ del total de infectados en el país), el grupo $C$ con el municipio que ocupó la posición 193 y el grupo D con el municipio que ocupó la posición 402. Estos grupos reflejan las diversas dinámicas específicas observadas en Colombia y en su conjunto son un reflejo de la totalidad de los municipios del país (cuadro 1).

Así, con estos cuatro grupos se construyó un espacio general que cubría todo el espectro posible de niveles de incidencia de malaria y se llegó a una generalización ajustada a la ley de la probabilidad y la entropía no equiprobable, que permitiría hacer predicciones, tanto generales como específicas para cualquier caso particular.

Con el fin de simplificar la dinámica estudiada y poder aplicar la teoría de las probabilidades - lo que facilitaría el estudio predictivo del fenómeno-, además de agrupar los eventos probabilísticos que correspondían a los brotes, se representaron los eventos probabilísticos que correspondían a la dinámica normal de la enfermedad (ausencia de brotes). Para ello se definieron rangos epidemiológicos, definidos por los múltiplos de 50 casos para los municipios del grupo $\mathrm{A}$, de 25 casos para los del grupo B, de 10 
para los del grupo C y de 5 para los del grupo D. Este agrupamiento se basó en los cambios semanales en el número de infectados en esos municipios en los últimos cinco años.

\section{Cálculos básicos del método}

Se calculó un valor de probabilidad en el que cada rango epidemiológico se consideró como un posible evento. Este valor cuantifica la probabilidad de que en una semana epidemiológica específica se registre un número dado de casos, expresado mediante un rango epidemiológico determinado. Para ello se calcularon los valores de probabilidad específica o secuencia anual (P) para cada combinación anual de rangos epidemiológicos en cada municipio a partir de la siguiente fórmula:

$$
\mathrm{P}=\prod_{i=1}^{n}(\mathrm{P} i)^{j}
$$

Ecuación 1

donde, $\mathrm{P} i$ es el valor de la probabilidad para el rango epidemiológico $i$, es decir la frecuencia de aparición del rango epidemiológico $i$ dividido entre la suma de todas las frecuencias de rangos epidemiológicos para cada año; $j$ es el número de apariciones del rango epidemiológico $i$ (la suma de todas las frecuencias de aparición debe ser igual a 53, que es el número total de semanas epidemiológicas en un año). El valor de $i$ puede variar desde 1, que es el valor de probabilidad para el rango epidemiológico 1, hasta $n$, que corresponde al valor de probabilidad para el rango epidemiológico mayor.

Con el fin de acotar la dinámica anual para cada año y cada municipio, las 53 semanas se agregaron en grupos de tres semanas consecutivas solapadas, es decir, se agruparon las semanas 1-3, luego las semanas $2-4$ y así sucesivamente hasta las semanas 51-53. Este agrupamiento demostró ser el que mejor porcentaje de acierto de las predicciones ofrecía con respecto a los datos reales, además de permitir contrastar la dinámica en períodos cortos y comparables con lo sucedido previamente. El valor de la probabilidad para cada grupo de semanas se calculó mediante la multiplicación de las probabilidades asociadas con cada semana. Posteriormente se calculó el valor de la entropía (S), según Boltzmann-Gibbs, para cada grupo de semanas, año y municipio, mediante la siguiente ecuación:

$$
\mathrm{S}=-\mathrm{k} \sum_{i=1}^{n} \mathrm{P}\left(\mathrm{A}_{i}\right) \times \operatorname{LnP}\left(\mathrm{A}_{i}\right) \quad \text { Ecuación } 2
$$

donde, $i$ es la semana estudiada; $\mathrm{P}\left(\mathrm{A}_{i}\right)$ es la probabilidad del rango epidemiológico presente en la semana $i$; y $\mathrm{k}$ es la constante de Boltzmann, igual a 1,38 x $10^{-23}$ joules/grados kelvin.

A partir de la ecuación anterior se pasó la constante de Boltzmann al miembro izquierdo de la ecuación para calcular el valor de la relación S/k:

$$
\frac{\mathrm{S}}{\mathrm{k}}=-\sum_{i=1}^{n} \mathrm{P}\left(\mathrm{A}_{i}\right) \times \operatorname{LnP}\left(\mathrm{A}_{i}\right) \quad \text { Ecuación } 3
$$

El valor de la relación $\mathrm{S} / \mathrm{k}$ permite predecir cambios en la dinámica de la incidencia de malaria en los grupos de tres semanas para cada municipio.

\section{Análisis físico y matemático y confirmación de las dinámicas}

A partir de los valores de la relación $\mathrm{S} / \mathrm{k}$ calculados para cada grupo de tres semanas de cada año estudiado y los $10 \mathrm{mu}$ nicipios analizados se obtuvieron restas consecutivas de la relación $\mathrm{S} / \mathrm{k}$ correspondiente a un grupo de tres semanas con respecto al valor obtenido para el grupo anterior. Estas restas se utilizaron para establecer las relaciones matemáticas en la dinámica de la malaria para los períodos de incidencia normal y los brotes.

Con las relaciones matemáticas encontradas se establecieron los valores límites que se relacionaban con cambios en la dinámica de casos, ya sea un aumento o una disminución. Estos valores se utilizaron para predecir los cambios en la dinámica de casos - en particular los brotes- en los 810 municipios del país en el período de 2003 a 2007. ${ }^{2} \mathrm{Al}$ final, se compararon las predicciones con los datos epidemiológicos reales.

\section{RESULTADOS}

El número de casos de malaria notificados en los 10 municipios seleccionados

\footnotetext{
2 Los resultados correspondientes a los 810 municipios de Colombia no se presentan por razones de espacio, pero están disponibles mediante solicitud al autor.
}

varió entre un mínimo de 0 (rango epidemiológico 1) y un máximo de 609 (rango epidemiológico 13), este último registrado en el municipio Tierralta, departamento de Córdoba, en la semana epidemiológica 4 de 2007 (cuadro 1).

Los valores de probabilidad para los grupos de municipios variaron entre 0,019 y 1,00 (cuadro 2), mientras que los valores de probabilidad específica para cada combinación anual de rangos epidemiológicos fluctuaron entre $9,35 \times 10^{-53}$ y 1,00 (cuadro 3), con diferencias entre los grupos de municipios estudiados.

La campaña antimalárica realizada por el Instituto Nacional de Salud en el municipio Buenaventura, departamento del Valle del Cauca, en los años 2006-2007 se reflejó en los menores valores de incidencia anual durante los dos últimos años con respecto tanto a los años anteriores como a los otros municipios de su grupo. Esta intervención provocó cambios en la dinámica de la enfermedad y generó una mayor equiprobabilidad al mantener rangos epidemiológicos más bajos.

Los valores de probabilidad calculados para cada grupo de tres semanas durante el período 2003-2007 en cada municipio variaron entre $2,01 \times 10^{-05}$ y 1,00 . El valor de la entropía calculada según Boltzmann-Gibbs para todos los municipios en cada año, a partir de los valores de probabilidad de los grupos de tres semanas, varió entre 0 y $1,52 \times 10^{-23}$. El valor obtenido para la relación $\mathrm{S} / \mathrm{k}$ calculada a partir de la probabilidad de los grupos de tres semanas para cada municipio varió entre 0,31 y 1,10, con los mayores valores en el municipio Turbo en 2005 (cuadro 4). El valor de la diferencia entre el valor de la relación $S / k$ de un grupo de semanas y el valor correspondiente al grupo anterior en cada municipio varió entre $-0,23$ y 0,29 (cuadro 4).

Las restas de la relación $\mathrm{S} / \mathrm{k}$ de los grupos de tres semanas consecutivas que no correspondían a brotes de malaria resultaron negativas, como se observa en el municipio Buenaventura en el grupo de semanas 10-12 de $2005(-0,03)$. Sin embargo, en el grupo de las semanas 11-13 - que contiene la semana 13, que presentó un elevado número de casos-, esa resta fue de 0,20 (cuadro 4) y la siguiente resta consecutiva para el grupo de las semanas 12-14, aunque menor, permaneció positiva, lo que indica que en ese grupo de semanas se presentó un mayor número de casos que en el grupo de las semanas 11-13. Más adelante, el 
CUADRO 1. Comportamiento (números mínimo y máximo semanal y total anual) de los casos de malaria en los municipios seleccionados, agrupados según el número de casos anuales, Colombia, 2003-2007

\begin{tabular}{|c|c|c|c|c|c|c|c|c|c|c|c|c|c|c|c|c|}
\hline \multirow[b]{2}{*}{ Grupo ${ }^{a} /$ municipio } & \multicolumn{3}{|c|}{2003} & \multicolumn{3}{|c|}{2004} & \multicolumn{3}{|c|}{2005} & \multicolumn{3}{|c|}{2006} & \multicolumn{3}{|c|}{2007} & \multirow{2}{*}{$\begin{array}{c}\text { Total } \\
2003- \\
2007\end{array}$} \\
\hline & Mínimo & Máximo & Total & Mínimo & Máximo & Total & Mínimo & Máximo & Total & Mínimo & Máximo & Total & Mínimo & Máximo & Total & \\
\hline \multicolumn{17}{|l|}{ A } \\
\hline Tierralta & 16 & 391 & 13940 & 49 & 561 & 17640 & 91 & 454 & 11813 & 4 & 447 & 9362 & 7 & 609 & 14938 & 67693 \\
\hline Montelíbano & 1 & 257 & 7094 & 2 & 258 & 6618 & 57 & 219 & 6388 & 32 & 277 & 7440 & 4 & 375 & 9147 & 36687 \\
\hline Puerto Libertador & 4 & 367 & 9101 & 2 & 291 & 7465 & 1 & 299 & 7561 & 1 & 296 & 2337 & 3 & 606 & 8312 & 34776 \\
\hline Cáceres & 1 & 160 & 2457 & 1 & 254 & 5798 & 1 & 272 & 4400 & 1 & 126 & 3001 & 1 & 156 & 3248 & 18904 \\
\hline Turbo & 0 & 186 & 2987 & 1 & 113 & 2396 & 1 & 144 & 2952 & 5 & 149 & 4611 & 12 & 212 & 5616 & 18562 \\
\hline Buenaventura & 2 & 173 & 2586 & 32 & 408 & 7335 & 8 & 271 & 6001 & 1 & 71 & 1080 & 0 & 45 & 752 & 17754 \\
\hline \multicolumn{17}{|l|}{$\mathrm{B}$} \\
\hline Taraza & 0 & 134 & 2451 & 1 & 108 & 1962 & 3 & 148 & 2281 & 2 & 209 & 3767 & 1 & 176 & 2939 & 13400 \\
\hline Necoclí & 1 & 121 & 2721 & 1 & 111 & 2892 & 1 & 116 & 2716 & 1 & 97 & 2200 & 1 & 80 & 1477 & 12006 \\
\hline \multicolumn{17}{|l|}{ C } \\
\hline Tuluá & 0 & 3 & 34 & 0 & 5 & 30 & 0 & 3 & 24 & 0 & 2 & 17 & 0 & 3 & 14 & 119 \\
\hline \multicolumn{17}{|l|}{$\mathrm{D}$} \\
\hline Los Palmitos & 0 & 1 & 1 & 0 & 1 & 1 & 0 & 2 & 4 & 0 & 3 & 8 & 0 & 3 & 14 & 28 \\
\hline
\end{tabular}

a Los grupos se formaron por la posición que ocupaba el municipio según el número de casos de malaria durante el período $2003-2007$ entre los 820 municipios de Colombia. Grupo A: los seis municipios con mayor número de casos anuales; grupo B: los municipios que ocuparon las posiciones 10 y 11 ; grupo C: el municipio que ocupó la posición 193; y grupo D: el municipio que ocupó la posición 402.

valor de la resta para el grupo de las semanas 13-15 fue de 0,20, lo que refleja que en ese período hubo un aumento en el número de casos del brote que empezó en la semana 13 y terminó en la semana 18. El valor de cero en los grupos de semanas 14-16 y 15-17 muestra una estabilización en el número de casos con respecto a las semanas anteriores, lo que confirmó la presencia de un brote. Este comportamiento se mantiene hasta el grupo de las semanas 16-18, cuando la resta vuelve a ser negativa $(-0,20)$ e indica el fin del brote y el regreso paulatino al estado "normal".

De esta forma, se puede establecer que las relaciones para determinar que la dinámica de casos corresponde a un brote son las siguientes:

1. Cuando la resta de la relación $S / k$ se encuentra entre 0,12 y 0,29, como se observó, respectivamente, en el municipio Turbo, del departamento Antioquia, en la semana 39 de 2005 con 131 casos y en el municipio Cáceres, del departamento Antioquia, en el grupo de semanas 23-25 de 2003 con 110 casos en la semana 25 (cuadro 4). En el caso de Cáceres, el valor de -0,06 (que aunque negativo es muy cercano a cero) indica la estabilización de la dinámica, mientras que el valor $-0,23$ indica el fin del pico en la semana 28, con solamente 49 infectados. Valores altos de la relación $\mathrm{S} / \mathrm{k}$ no indican necesariamente que ocurre un brote, pues estos pueden aparecer con pocos casos semanales.
Por ejemplo, aunque en la semana 34 de 2006 se notificaron 96 casos en Tierralta, la resta de las relaciones $\mathrm{S} / \mathrm{k}$ en las semanas 32-34 fue de 0,19, por lo que no se consideró un brote. Esto se confirma al comparar la relación $\mathrm{S} / \mathrm{k}$ de ese grupo de semanas $(0,19)$ con el número de casos de la semana 36 (269), cuando sí se declaró un brote y la relación $\mathrm{S} / \mathrm{k}$ fue de 0,13 (cuadro 4).

2. Cuando la resta de la relación $S / k$ de un grupo de semanas con respecto a otro que corresponde a un brote es cero o mayor. Esto puede indicar que se mantiene la dinámica de casos característica de un brote. Por ejemplo, la resta de las relaciones $\mathrm{S} / \mathrm{k}$ del grupo de las semanas 14-16 de 2005 en el municipio Buenaventura fue de cero, pero como esta resta se hizo con respecto al grupo de las semanas 13-15, que contienen un brote, se debe concluir que este se mantiene en el grupo de semanas 14-16 (cuadro 4).

3. Cuando se presentan más de cuatro restas consecutivas de la relación $\mathrm{S} / \mathrm{k}$ igual a 0,08 o mayor con respecto a semanas que presentan un alto número de casos. Este comportamiento se encontró en el municipio Buenaventura en el grupo de las semanas 19-23 de 2004 (cuadro 4).

Se encontró que los brotes ocurridos en los 10 municipios estudiados se asociaron con al menos una de estas tres relaciones. Esto se confirmó con los datos de los 810 municipios de Colombia entre
2003 y 2007. En contraposición, en 99,86\% de los grupos de semanas estudiadas con una dinámica normal de casos no se cumplió ninguna de estas relaciones.

\section{DISCUSIÓN}

En este trabajo se presenta el primer método de predicción de brotes de malaria en Colombia a partir de la cuantificación probabilística del número de enfermos - agrupados en rangos epidemiológicos- y la evaluación de su distribución espacio-temporal no equiprobable para cada año. Este método se basa en las probabilidades calculadas para grupos de tres semanas consecutivas y su evaluación según la ley de la entropía y las restas de la relación $\mathrm{S} / \mathrm{k}$. La simplificación del número de casos en rangos epidemiológicos se basa en las leyes de la probabilidad y la entropía y permite abordar la dinámica de casos de malaria desde la visión de la mecánica estadística.

Así, la dinámica de casos se caracteriza mediante relaciones cuantitativas y los brotes se identifican cuando esa relación cumple determinadas premisas. Esto permite hacer predicciones de la aparición de un número de casos equivalente a un brote para cada municipio y momento específicos a partir de los datos de períodos de tiempo cortos (tres semanas). Este método se puede utilizar en sistemas de vigilancia y alerta temprana de brotes de malaria en Colombia a partir de valores discretos que agrupan un número determinado de casos. 


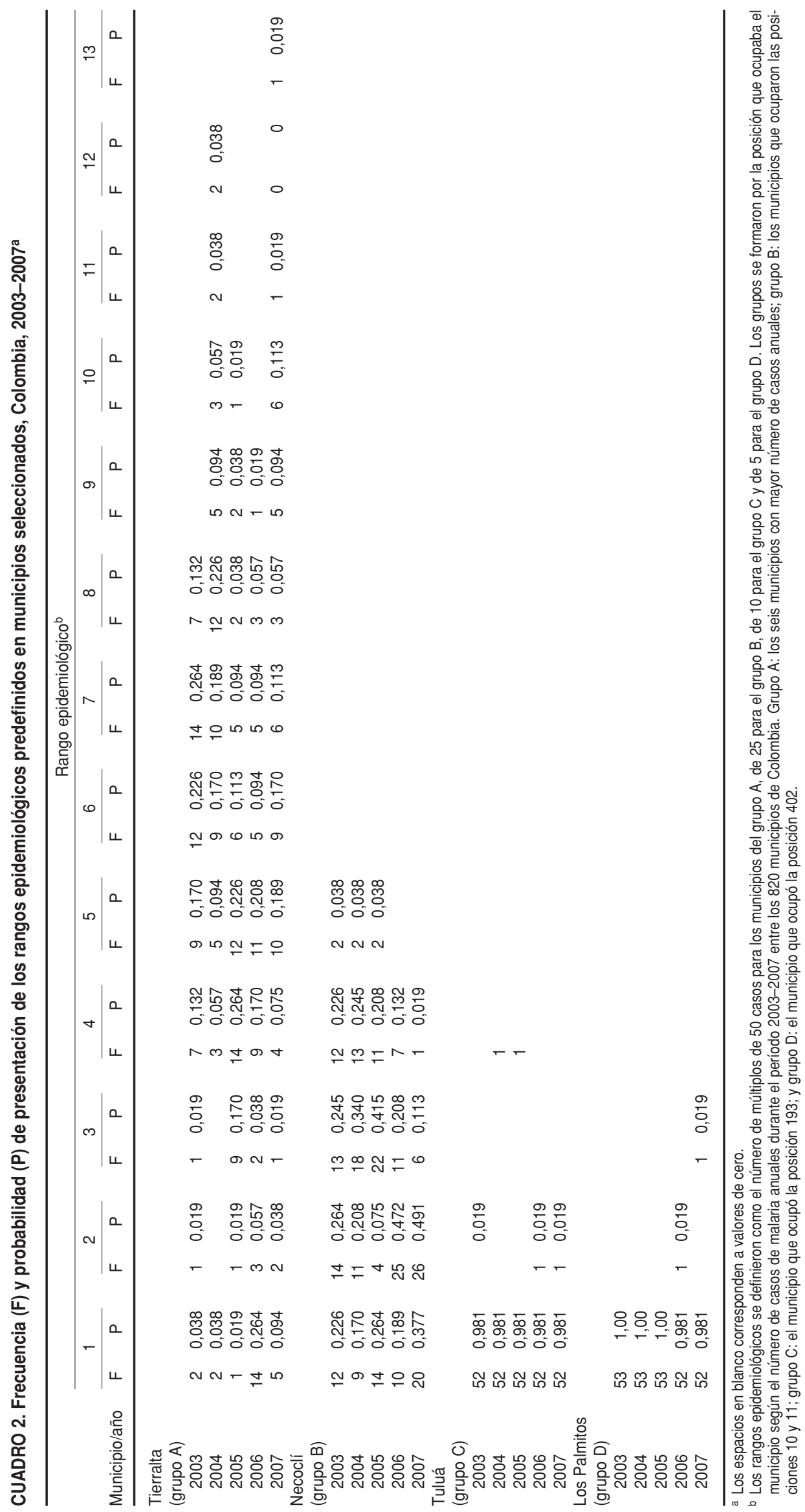


CUADRO 3. Valores de probabilidad específica o secuencia anual para cada combinación anual de rangos epidemiológicos predefinidos, Colombia, 2003-2007

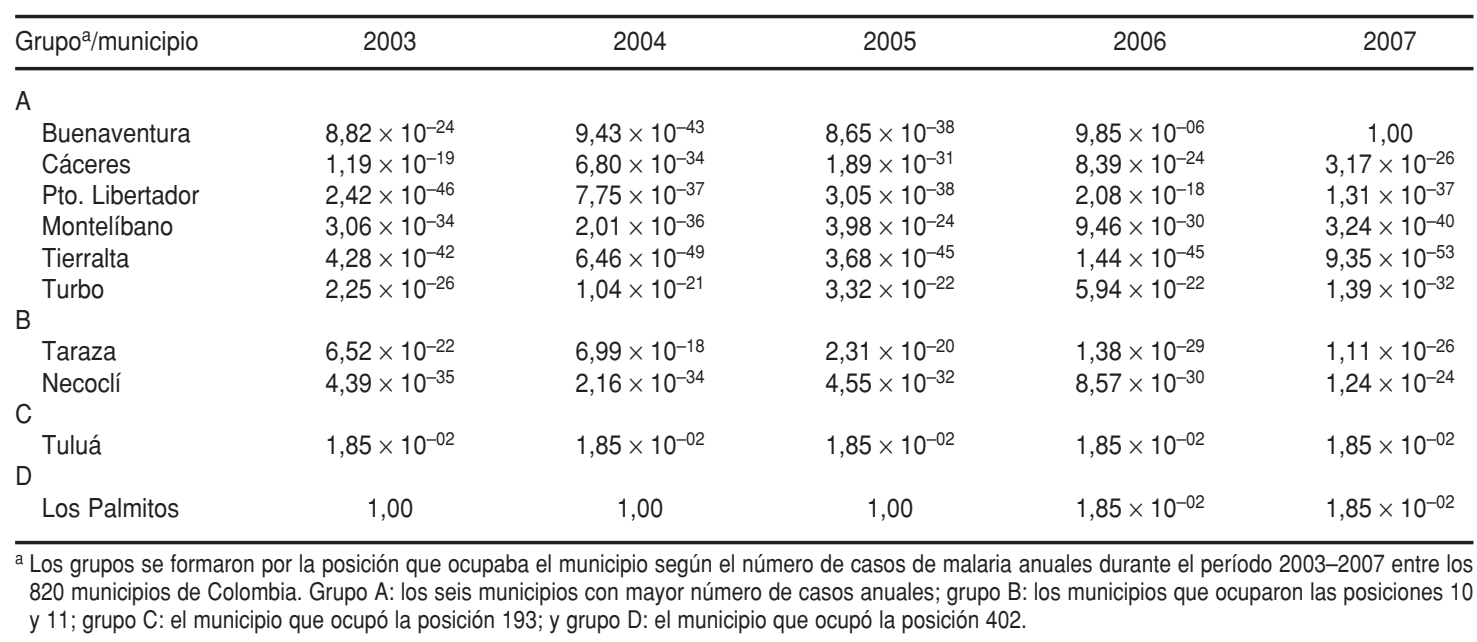

CUADRO 4. Número semanal de casos de malaria y valores de la relación $\mathrm{S} / \mathrm{k}$ y sus diferencias (restas) entre los grupos de tres semanas solapadas en siete municipios escogidos, Colombia, 2003-2007

\begin{tabular}{|c|c|c|c|c|c|c|c|c|c|}
\hline \multirow{2}{*}{$\frac{\text { Municipio/año/indicador }}{\text { Turbo, } 2005}$} & \multicolumn{9}{|c|}{ Valor obtenido en semanas seleccionadas ${ }^{a}$} \\
\hline & & & & & & & & & \\
\hline Semana & 33 & 34 & 35 & 36 & 37 & 38 & 39 & 40 & 41 \\
\hline Casos notificados & 83 & 53 & 81 & 48 & 83 & 3 & 131 & 66 & 50 \\
\hline $\mathrm{S} / \mathrm{k}^{\mathrm{b}}$ & & & 1,10 & 1,07 & 1,07 & 1,05 & 0,93 & 0,93 & 0,93 \\
\hline Resta $^{\mathrm{C}}$ & & & & 0,02 & 0 & 0,02 & $\underline{0,12}$ & 0 & 0 \\
\hline \multicolumn{10}{|l|}{ Cáceres, 2003} \\
\hline Semana & 22 & 23 & 24 & 25 & 26 & 27 & 28 & 29 & 30 \\
\hline Casos notificados & 60 & 32 & 35 & 110 & 43 & 91 & 49 & 160 & 36 \\
\hline $\mathrm{S} / \mathrm{k}$ & & & 0,98 & 0,68 & 0,68 & 0,75 & 0,98 & 0,75 & 0,68 \\
\hline Resta & & & & $\underline{0,29}$ & 0 & $-0,06$ & $-0,23$ & $\underline{0,23}$ & 0,06 \\
\hline \multicolumn{10}{|l|}{ Tierralta, 2006} \\
\hline Semana & 30 & 31 & 32 & 33 & 34 & 35 & 36 & 37 & 38 \\
\hline Casos notificados & 4 & 37 & 35 & 35 & 96 & 168 & 269 & 121 & 247 \\
\hline $\mathrm{S} / \mathrm{k}$ & & & 1,05 & 1,06 & 0,87 & 0,82 & 0,69 & 0,65 & 0,67 \\
\hline Resta & & & & 0 & 0,19 & $\underline{0,05}$ & 0,13 & 0,04 & $-0,03$ \\
\hline \multicolumn{10}{|l|}{ Buenaventura, 2004} \\
\hline Semana & 16 & 17 & 18 & 19 & 20 & 21 & 22 & 23 & 24 \\
\hline Casos notificados & 166 & 158 & 195 & 234 & 202 & 343 & 354 & 408 & 301 \\
\hline $\mathrm{S} / \mathrm{k}$ & & & 0,74 & 0,66 & 0,57 & 0,49 & 0,40 & 0,31 & 0,31 \\
\hline Resta & & & & $\underline{0,08}$ & $\underline{0,08}$ & $\underline{0,08}$ & 0,09 & 0,09 & 0 \\
\hline \multicolumn{10}{|l|}{ Buenaventura, 2005} \\
\hline Semana & 10 & 11 & 12 & 13 & 14 & 15 & 16 & 17 & 18 \\
\hline Casos notificados & 130 & 178 & 113 & 271 & 231 & 264 & 252 & 210 & 118 \\
\hline $\mathrm{S} / \mathrm{k}$ & & 0,97 & 1,00 & 0,80 & 0,69 & 0,49 & 0,49 & 0,49 & 0,69 \\
\hline Resta & & & $-0,03$ & $\underline{0,20}$ & $\underline{0,11}$ & 0,20 & 0 & 0 & $-0,20$ \\
\hline
\end{tabular}

La amplia variación observada en los valores de probabilidad específica para cada combinación anual de rangos epidemiológicos (entre 9,35 × $10^{-53}$ y 1,00) demuestra la dificultad de predecir la dinámica de esta enfermedad a partir de los datos de años anteriores.

Si bien se han desarrollado modelos de corredores endémicos para predecir la aparición de brotes de malaria (3), estos se basan en una media central y un recorrido de fluctuaciones normales de la dinámica para cada uno de los meses, calculados a partir de series de casos notificados de 5-7 años. Una premisa para el uso de estos modelos es que el comportamiento de la dinámica de casos de malaria de cada mes debe ser similar a lo registrado en el mismo mes de años anteriores. Sin embargo esto no ocurre así y la comparación temporal retroactiva del número de casos dentro de un sistema de vigilancia se hace muy difícil. Además, pueden ocurrir cambios en el comportamiento de los brotes, los sistemas de vigilancia, las decisiones de salud pública y la dispersión de los casos notificados.

Algunos autores han planteado que el azar desempeña un papel fundamental en la aplicación de otros modelos 
—incluido el de corredores endémicos-, por lo que se hace necesario aplicar funciones logarítmicas a los datos reales para poder estudiar el comportamiento estadístico epidemiológico de la dinámica de casos (3). De la misma forma se ha encontrado que en zonas pequeñas o de pocos casos, el papel del azar es más prominente y se dificulta más el uso de esos modelos epidemiológicos. Una forma de resolver este problema es ampliar la zona o el período de estudio (3). Sin embargo, esa ampliación conllevaría un menor detalle de los datos y una pérdida de eficiencia en las decisiones de salud pública. Por el contrario, en la metodología propuesta aquí no se toma en cuenta el azar, pues el enfoque probabilístico permite cuantificar la dinámica de brotes a partir de las relaciones de la entropía para intervalos de tres semanas consecutivas sin importar la zona geográfica. Esto hace innecesario ampliar el período planteado inicialmente de una semana y demuestra la aplicabilidad efectiva del método, incluso para enfermedades raras o zonas de baja incidencia.

Otros modelos matemáticos buscan establecer relaciones entre algunos factores - como la temperatura, los regímenes de lluvia y otros factores climáticos, así como las relaciones de las poblaciones humanas con el vector, entre otros- y la dinámica del número de casos de diversas enfermedades (4-11, 17-20). Ese enfoque, que busca relaciones causales, no ha logrado establecer y cuantificar la relación entre estos factores y los cambios en la dinámica de las enfermedades. A diferencia de esos modelos, la metodología propuesta aquí se basa en leyes físicas y matemáticas que permiten abordar este problema y establecer valores cuantitativos predictivos desde una perspectiva acausal. De la misma manera que en la física teórica actual no se conciben causas que determinen el comportamiento de los fenómenos, sino órdenes matemáticos que permiten comprender su naturaleza (21, 22), el enfoque que predominó en este estudio fue que los fenómenos funcionan de una forma probabilística intencionada (como un dado "cargado"). Este enfoque - que media entre la idea de Einstein de que "Dios no juega a los dados" y la de Bohr acerca de que "el universo es probabilístico e indeterminado" (23) — contiene simultáneamente componentes de determinación e indeterminación en la predicción de la dinámica de casos y la aparición de brotes de malaria, lo que resuelve el problema de las fluctuaciones azarosas y permite predecir la forma "inercial" de la dinámica de la enfermedad.

Para Einstein el método físico teórico consta de dos partes: primero, descubrir los principios generales de la naturaleza a partir de la percepción de hechos particulares, y segundo, deducir a partir de estos principios las conclusiones (24). Similar a lo planteado por Einstein, en este trabajo se parte de las dinámicas particulares de 10 municipios -observada a partir de las leyes de la probabilidad y la entropía- para llegar a una generalización que permite explicar cualquier dinámica en cualquier municipio y predecir la aparición o no de brotes según la conservación o la variación de las probabilidad de que aparezca un número de casos.

Por otra parte, aunque se ha planteado que los modelos complejos son los más precisos en el estudio de la dinámica de casos de malaria (3), la metodología presentada aquí, aunque parte de conceptos complejos de probabilidad y entropía, es simple y en la práctica se basa en el número de casos y la resta de los valores de la relación $\mathrm{S} / \mathrm{k}$.

Al analizar estos resultados se debe tener presente que este trabajo se basó en los registros del Instituto Nacional de Salud de Colombia, que no pueden considerarse totalmente exhaustivos. Sin embargo, este es un problema que comparte cualquier otro modelo desarrollado.

Se puede concluir que la metodología desarrollada permite predecir la dinámica de los casos y los brotes de malaria en los municipios de Colombia, a partir de una inducción probabilística acausal realizada en base a las leyes de la probabilidad y la entropía.

Estos resultados son útiles para la planificación de intervenciones y políticas de salud pública, ya que brindan un mayor rigor y permiten alcanzar una mayor eficacia de una forma más simple que los métodos actualmente utilizados.

Se recomienda desarrollar, validar e implementar un programa informático que permita automatizar los cálculos de esta metodología para poder extender de forma sencilla y práctica su aplicación en la toma de decisiones y la vigilancia epidemiológica. Esta metodología debe validarse para su aplicación en la vigilancia de otras enfermedades, como el dengue.

Agradecimientos. Al Instituto Nacional de Salud de Colombia, en particular a Salua Osorio y Viviana Cerón, por facilitar los datos del programa SIVIGILA y su apoyo a esta investigación, y a los integrantes del grupo de investigación Insight.

\section{REFERENCIAS}

1. World Health Organization. Data world malaria report. Geneva: WHO; 2008. Hallado en http://www.who.int/malaria/wmr2008/ malaria2008.pdf. Acceso el 14 de enero de 2009. (Documento WHO/HTM/GMP/2008.1.)

2. Instituto Nacional de Salud. Indicadores del estado de salud. Índice parasitario anual de malaria. Santa Fe de Bogotá: Así Vamos en Salud; 2009. Hallado en http://www. asivamosensalud.org/index.php?option= com content\&view $=$ article\&id $=93: \%$ C3\% 8 Dndice parasitario anual de malaria\&catid= 55:Indicadores\&Itemid=74. Acceso el 14 de enero de 2009.
3. Bortman M. Elaboración de corredores o canales endémicos mediante planillas de cálculo. Rev Panam Salud Publica. 1999;5(1):1-8.

4. Patz JA, Epstein PR, Burke TA, Balbus JM. Global climate change and emerging infectious diseases. J Am Med Assoc. 1996;275: 217-23.

5. Martens WJM, Jetten TH, Focks DA. Sensitivity of malaria, schistosomiasis and dengue to global warming. Clim Change. 1997;35: $145-56$.

6. Unninayar SS, Sprigg W. Climate and the emergence and spread of infectious diseases. Eos. 1995;76(47):478.
7. Epstein PR, Chikwenhere GP. Biodiversity questions [letter]. Science. 1994;265:1510-1.

8. Bouma MJ, van der Kaay HJ. Epidemic malaria in India and El Niño Southern Oscillation. Lancet. 1994;344:1638-9.

9. Bouma MJ, Dye C. Cycles of malaria associated with El Niño in Venezuela. J Am Med Assoc. 1997;278:1772-4.

10. Poveda G, Rojas W. Impacto del fenómeno de El Niño sobre la intensificación de la malaria en Colombia. Memorias del XII Seminario Nacional de Hidráulica e Hidrología. Santa Fe de Bogotá: Sociedad Colombiana de Ingenieros; 1996. 
11. Poveda G, Rojas W. Evidencias de la asociación entre brotes epidémicos de malaria en Colombia y el fenómeno El Niño - Oscilación del Sur. Rev Acad Colomb Cien. 1997; 21(81):421-9.

12. Rodríguez J, Correa C. Predicción temporal de la epidemia de dengue en Colombia: dinámica probabilista de la epidemia. Rev Salud Publica. 2009;11(3):443-53.

13. Feynman RP, Leighton RB, Sands M. Probabilidad. En: Feynman RP, Leighton RB, Sands M. Física. Vol. 1. Wilmington, WA: AddisonWesley Iberoamericana; 1964. Pp. 6.1-16.

14. Matvéev A. Física molecular. 1.a ed. Moscú: Mir; 1987.

15. Tolman R. Principles of statistical mechanics. 1st ed. New York: Dover Publications; 1979.

16. Shannon CE. The mathematical theory of communication. Bell Syst Tech J. 1948;27: 379-423.
17. Briët $\mathrm{O}$, Vounatsou $\mathrm{P}$, Gunawardena DM, Galappaththy GNL, Amerasinghe PH. Models for short term malaria prediction in Sri Lanka. Malar J. 2008;7:76

18. Jones A, Uddenfeldt $\mathrm{U}$, Morse AP, Hastings IM, Gagnon AS. Climate prediction of El Niño malaria epidemics in North-West Tanzania. Malar J. 2007;6:162.

19. Moreno JE, Rubio-Palis Y, Páez E, Pérez E, Sánchez V. Abundance, biting behavior and parous rate of anopheline mosquito species in relation to malaria incidence in gold-mining areas of southern Venezuela. Med Vet Entomol. 2007;21:339-49.

20. Wysea AP, Bevilacquaa L, Rafikovd M. Simulating malaria model for different treatment intensities in a variable environment. Ecol Modell. 2007;206:322-30

21. Peitgen $H$, Jürgens $H$, Saupe D. Strange attractors, the locus of chaos. En: Peitgen $\mathrm{H}$,
Jürgens H, Saupe D. Chaos and fractals. NewYork: Springer-Verlag; 1992. Pp. 655-768.

22. Feynman RP, Leighton RB, Sands M. Comportamiento cuántico. En: Feynman RP Leighton RB, Sands M. Física. Vol. 1. 1.a ed. México, D.F.: Wilmington Addison-Wesley Iberoamericana; 1998. Pp. 37.1-15.

23. Calude CS, Stay MA. From Heisenberg to Godel via Chaitin. Int J Theor Phys. 2007; 46(8):2013-25.

24. Einstein A. Principios de física teórica. En: Einstein A. Sobre la teoría de la relatividad y otras aportaciones científicas. Madrid: Sarpe; 1983. Pp. 29-32.

Manuscrito recibido el 21 de julio de 2009. Aceptado para publicación, tras revisión, el 12 de noviembre de 2009.

ABSTRACT Objective. To develop a methodology for forecasting the seasonal dynamic of malaria outbreaks in the municipalities of Colombia.

\section{A method for forecasting the seasonal dynamic of malaria in the municipalities of Colombia}

Key words
Methods. Epidemiologic ranges were defined by multiples of 50 cases for the six municipalities with the highest incidence, 25 cases for the municipalities that ranked 10th and 11th by incidence, 10 for the municipality that ranked 193rd, and 5 for the municipality that ranked 402nd. The specific probability values for each epidemiologic range appearing in each municipality, as well as the $S / \mathrm{k}$ value-the ratio between entropy $(S)$ and the Boltzmann constant $(\mathrm{k})$ - were calculated for each threeweek set, along with the differences in this ratio divided by the consecutive sets of weeks. These mathematical ratios were used to determine the values for forecasting the case dynamic, which were compared with the actual epidemiologic data from the period 2003-2007.

Results. The probability of the epidemiologic ranges appearing ranged from 0.019 and 1.00 , while the differences in the $S / \mathrm{k}$ ratio between the sets of consecutive weeks ranged from 0.23 to 0.29 . Three ratios were established to determine whether the dynamic corresponded to an outbreak. These ratios were corroborated with real epidemiological data from 810 Colombian municipalities.

Conclusions. This methodology allows us to forecast the malaria case dynamic and outbreaks in the municipalities of Colombia and can be used in planning interventions and public health policies.

Malaria; forecasting; temporal distribution; disease outbreaks; epidemiologic surveillance; Colombia. 